\title{
Supraclavicular lymphadenopathy as the initial presentation of metastatic prostate cancer: A case report and review of literature
}

\author{
Garson Chan, MD; $;^{*}$ Trustin Domes, MD, FRCSC ${ }^{\dagger}$ \\ *College of Medicine, University of Saskatchewan, Saskatoon, SK; 'Urologist, Saskatoon Health Region, Saskatoon, SK
}

Cite as: Can Urol Assoc J 2013;7(5-6):e433-5. http://dx.doi.org/10.5489/cuaj.1385 Published online June 12, 2013.

\begin{abstract}
Prostate cancer usually metastasizes to the regional lymph nodes, and distal metastases to supraclavicular lymph nodes are rarely reported, especially as an initial presentation. Limited case reports describe cervical lymphadenopathy as the initial presentation of metastatic prostate cancer, and often with widely disseminated disease. Patients with this initial presentation rarely undergo digital rectal examination or serum prostate-specific antigen (PSA) level measurement as part of their initial investigations. A high index of suspicion is necessary to make the diagnosis of prostate cancer in this clinical setting. We present a rare case of prostate carcinoma presenting with supraclavicular lymph node enlargement at the initial diagnosis. A review of the relevant literature is provided.
\end{abstract}

\section{Introduction}

Cancer of the prostate is one of the most common malignancies in men. Prostate cancer can present in various ways, including asymptomatically with prostate-specific antigen (PSA) levels, local invasion and regional lymph node involvement or metastasis with systemic symptoms. ${ }^{1}$ The most common sites for prostate cancer metastases are local lymph nodes, bones and lungs. ${ }^{1}$ Although cervical lymph nodes are a common site of metastasis for head and neck cancers, metastasis from the prostate is extremely rare. ${ }^{2}$

\section{Case report}

A 61-year-old male, previously healthy, was referred to thoracic surgery from his primary care provider; he presented with a 4-month history of a palpable painless left supraclavicular mass. In addition, he had some urinary symptoms with difficulty emptying, poor urinary stream, as well as some urgency which improved after starting both dutasteride and tamsulosin. He denied any bone pain or significant constitutional symptoms, and his medical and family history was unremarkable. A digital rectal examination (DRE) was not performed at either assessment.

Routine blood biochemistry was within the normal range, except for an elevated creatinine at $143 \mathrm{umol} / \mathrm{L}$ and a PSA of $16 \mathrm{ng} / \mathrm{mL}$. A fine needle biopsy of the left supraclavicular lymph node was performed. This initial biopsy (Fig. 1) showed adenocarcinoma of undetermined origin, but stained negative for the prostatic markers of PSA and prostate-specific acid phosphatase (PAP).

The patient subsequently underwent a computed tomography (CT) scan of the neck, chest and abdomen in search of the primary source (Fig. 2). The CT demonstrated adenopathy within the supraclavicular regions, retroperitoneum and pelvis. There was also evidence of a soft tissue mass from the prostate, emanating to the bladder and bilateral hydroureteronephrosis.

At this point, he was referred to urology for further evaluation. A DRE demonstrated a hard rocky prostate on the right side, consistent with clinical T3 disease.

Cystoscopy was performed, which revealed a large median lobe with an obstructing prostate and moderate trabeculation of the bladder. Around this time, the patient went into acute urinary retention and, because of his elevated creatinine, subsequently had a transurethral resection of his prostate. The specimen submitted from the prostate resection showed high-grade prostate cancer (Gleason score $4+5=9$ ). His voiding improved significantly after his prostate resection, his creatinine stabilized and his hydronephrosis resolved. A Tc-99m whole body bone scan was also completed which revealed no skeletal metastases.

To obtain a more adequate specimen for pathological determination, we performed an excisional biopsy of the left supraclavicular, which did stain positive with PAP and PSA (Fig. 3a, Fig. 3b). It stained weakly and focally with CK7 and CK20, synaptophysin. This specimen was consistent with metastatic prostate cancer.

The patient was initiated on hormone therapy with bicalutamide and leuprolide in the outpatient clinic and a 


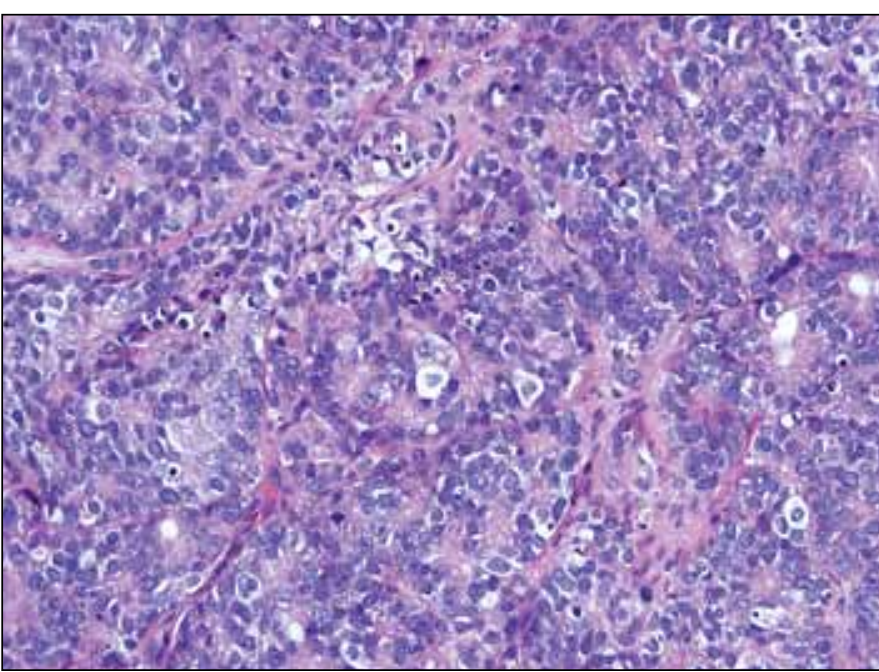

Fig. 1. Fine needle biopsy of the left neck mass showing adenocarcinoma of undetermined origin, comprised of malignant cells.

referral to the Saskatoon Cancer Centre was made. Several months later, the patient had relief of his voiding symptoms and his PSA decreased to undetectable levels. He remains clinically stable and asymptomatic on androgen blockade.

\section{Discussion}

Metastases from prostate cancer most commonly occurs via lymphatic spread to regional lymph nodes, direct invasion to the pelvic organs or systemically to the axial skeleton. ${ }^{1}$ The local growth of prostate cancer may cause symptoms, such as obstruction and irritation, when voiding. However, it usually presents with only voiding symptoms.

In regards to metastatic lymphatic spread of prostate cancer, there typically is a step-wise progression. This involves

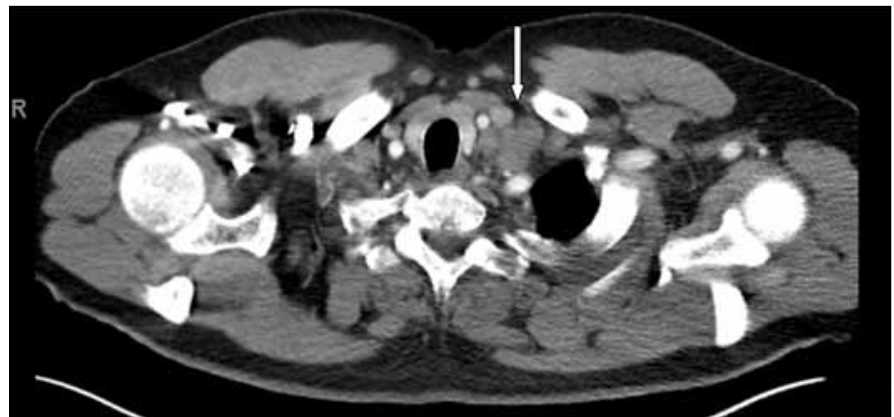

Fig. 2. Computed tomography scan of the neck demonstrating a cluster of enlarged lymph nodes involving the left supraclavicular fossa, measuring $3.5 \times 4.9 \mathrm{~cm}$ in diameter (arrow).

first the regional lymph nodes (obturator and pelvic) to the retroperitoneum, then cysterna chyli, and finally the thoracic duct where the lymphatic system enters the systemic circulation by the left subclavian vein. Left cervical nodes are near the entry of thoracic duct into the left subclavian vein which drains to the left jugular trunk. It is thought that cancer cells are then able to spread to these nodes via retrograde spread. ${ }^{3,4}$

Most tumours of the head and neck arise from primary cancers of skin, salivary glands, thyroid and upper gastrointestinal tract, with occasional metastases from the lung, kidney and breast. In most cases, prostate cancer was not considered in the differential diagnosis of supraclavicular lymphadenopathy at the initial presentation. As such, diagnoses of prostate cancer are often made only after biopsy of these lymph nodes confirmed with staining techniques for prostate-specific markers. In the literature, most of the cases of prostate cancer presenting with supraclavicular lymphadenopathy involved left-sided cervical lymphadenopathy (Virchow's Node), and only 1 case reported isolated

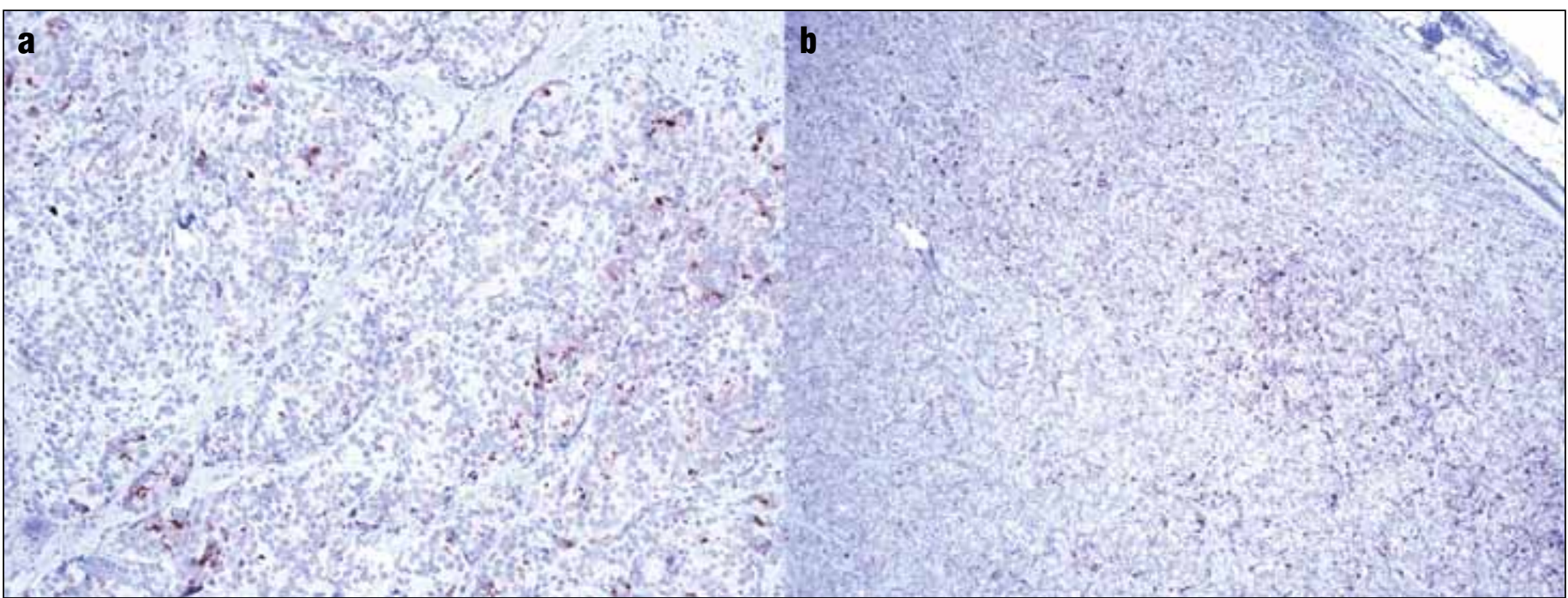

Fig. 3. Excisional biopsy of the left supraclavicular mass staining positive for prostate specific antigen (PSA) and prostate-specific acid phosphatase (PAP) in slides (a) and (b) respectively. 
right side involvement. ${ }^{5}$ Furthermore, the literature describes cases of metastatic supraclavicular lymphadenopathy with evidence of widespread systemic metastases or as the site of recurrence years later after treatment. Only 3 cases were reported that did not have widespread systemic metastases in addition to the supraclavicular lymphadenopathy. ${ }^{2,6}$ As such, it is rare to have distant metastasis to the supraclavicular lymph nodes without bone involvement or widespread systemic metastasis. This is only the third case reported with such a relatively low PSA $(<20 \mathrm{ng} / \mathrm{mL})$ for amount of lymph nodal metastasis present. ${ }^{2}$ However, even though the two previous cases had PSAs of $9.1 \mathrm{ng} / \mathrm{mL}$ and $14 \mathrm{ng} / \mathrm{mL}$, they had evidence of widespread systemic metastasis at the time of diagnosis. ${ }^{2}$ Most of the other cases reported had PSA levels in excess of $20 \mathrm{ng} / \mathrm{mL}$. In addition, most patients were older males (age $>65$ ), except one 42-year-old male with a high PSA of $153 \mathrm{ng} / \mathrm{mL}$ and widespread systemic metastasis in addition to his clinical supraclavicular lymphadenopathy. ${ }^{7}$

Butler and colleagues were among the first to describe a large number of cases $(n=19)$ that presented with supraclavicular lymphadenopathy as the initial presentation of metastatic prostate carcinoma. ${ }^{8}$ From the 19 cases observed, $42 \%$ had an abnormal DRE and $58 \%$ had a normal bone scan. Another case series evaluating men with metastatic prostate cancer included 15/26 cases that involved the supraclavicular lymph nodes; in this series, 58\% had an abnormal DRE, and $65 \%$ had an abnormal bone scan. ${ }^{9}$

It is important to have a high index of suspicion for metastatic prostate cancer in men presenting with clinical supraclavicular lymphadenopathy. As in this case, special staining with prostate cancer markers, like PSA and prostate-specific acid phosphatase (PAP), is important to diagnose or rule out metastatic prostate cancer - even if these men have no other manifestations of metastatic prostate cancer..$^{9,10}$

\section{Conclusion}

This case emphasizes the importance of considering prostate cancer in the differential diagnosis of males with supracla- vicular lymphadenopathy. Routine DRE and serum PSA levels are relatively easy and non-invasive procedures. In addition, these procedures should be done on all male patients with pathological persistent supraclavicular lymphadenopathy at the time of initial presentation. It is important to establish a diagnosis of metastatic prostate cancer early as anti-androgen treatment is available which can delay the progression of symptomatic metastases that may develop.

Competing interests: None declared.

This paper has been peer-reviewed.

\section{References}

1. Epstein Jl. Pathology of prostatic neoplasia. In: Wein AJ, Kavoussi LR, Novick AC, Partin AW, Peters CA. Campbell-Walsh Urology. 9th ed. Philadelphia: W.B. Saunders; 2007:2874-5.

2. Hematpour K, Bennett CJ, Rogers D, et al. Supraclavicular lymph node: incidence of unsuspected metastatic prostate cancer. Eur Arch Otorhinolaryngol 2006;263:872-4. hitp://dx.doi.org/10.1007/s00405-006-0066-2

3. Jones H, Anthony PP. Metastatic prostatic carcinoma presenting as left-sided cervical lymphadenopathy: a series of 11 cases. Histopathology 1992;21:149-54. http://dx.doi.org/10.1111/i.1365-2559.1992.tb00363.x

4. Wang HJ, Chiang PH, Peng JP, et al. Presentation of prostate carcinoma with cervical lymphadenopathy: report of three cases. Chang Gung Medical J 2004;27:840-4.

5. Ozgur A, llker Y, Turkeri LN. Cervical lymph node enlargement on the right side as the initial manifestation of metastatic prostate cancer. Arch Esp Urol 2003;56:859-61.

6. Carleton J, van der Riet P, Dahm P. Metastatic prostate cancer presenting as an asymptomatic neck mass. Prostate Cancer Prostatic Dis 2005;8:293-5. http://dx.doi.org/10.1038/si.pcan.4500805

7. Lin, YY, Lin DS, Kang BH, et al. Neck mass as the first presentation of metastatic prostatic adenocarcinoma. J Chin Med Assoc 2011;74:570-3. http://dx.doi.org/10.1016/i.jma.2011.09.012

8. Butler JJ, Howe C, Johnson D. Enlargement of the supraclavicular lymph nodes as the initial sign of prostatic carcinoma. Cancer 1971;27:1055-63. http://dx.doi.org/10.1002/1097-0142(197105)27:5<1055::AID-CNCR2820270509>3.0.C0;2-T

9. Cho KR, Epstein Jl. Metastatic prostatic carcinoma to supradiaphragmatic lymph nodes. A clinicopathologic and immunohistochemical study. Am J Surg Pathol 1987;11:457-63. http://dx.doi.org/10.1097/00000478-198706000-00006

10. Woo $K$, Weiczorek $R$, Torre $P$, et al. Prostate adenocarcinoma presenting as a large supraclavicular mass. Rev Urol 2001;3:102-5.

Correspondence: Dr. Trustin Domes, Saskatoon Health Region, 537 Medical Arts Building 750 Spadina Crescent East Saskatoon, SK S7K 3H3; trustindomes@gmail.com 